\title{
DYING IN A DGH
}

Tamsin Cargill, Matthew Turp, Jane Wale. Milton Keynes General Hospital, Milton Keynes, Milton Keynes

10.1136/bmjspcare-2014-000654.180

Background In Milton Keynes half of people die in hospital. It is not known how many patients and their families have the opportunity to discuss end-of-life care in hospital.

Methods Review of the electronic medical records of all those that died in Milton Keynes Hospital during November 2012.

Findings Of the 67 records analysed cancer, chronic obstructive pulmonary disease or left ventricular failure was the primary or secondary cause of death in $62 \% \quad(n=42) .31 \%$ of patients $(n=21)$ were seen by the palliative care team. Patients seen by palliative care were significantly more likely to have a signed Do Not Attempt Resuscitation form (95\%; $\mathrm{n}=20$ vs $78 \%$; $\mathrm{n}=36$; $\mathrm{p}=0.004)$, a discussion about end-of-life treatment $(\mathrm{n}=4 ; 19 \%$ vs. $n=2 ; 4 \% ; p=0.003$ ), and more likely to state their preferred place of death $(n=7 ; 33 \%$ vs. $n=2 ; 4 \% ; p=0.001)$. End-of-life issues were often discussed with the family rather than the patient themselves. End-of-life was discussed with the families of patients seen by palliative care in $90 \%$ of cases $(n=19)$ compared to $54 \%(n=25)$ of those not seen by palliative care.

Conclusions The end-of-life wishes were not discussed in all patients.All aspects of end of life care were significantly more likely to be discussed with patients or families who had been 
seen by the palliative care team. End-of- life care decisions were discussed with the patient's family more often than the patient themselves. Often patients were too unwell during their last illness to meaningfully participate in end of life discussions.

The AMBER initiative, soon to be piloted in Milton Keynes, promotes early identification of patients at risk of dying within 2-3 months and initiates end-of life care discussions with them. This and improved communication between primary and secondary care about a patient's end-of- life preferences should improve future care. 